\title{
BMJ Open Association of magnesium intake with type 2 diabetes and total stroke: an updated systematic review and meta- analysis
}

\author{
Binghao Zhao, ${ }^{1}$ Lianli Zeng, ${ }^{2}$ Jiani Zhao, ${ }^{2}$ Qian $\mathrm{Wu},{ }^{2}$ Yifei Dong, ${ }^{2}$ Fang Zou, ${ }^{3}$ \\ Li Gan, ${ }^{4}$ Yiping Wei, ${ }^{1}$ Wenxiong Zhang (D) ${ }^{1}$
}

To cite: Zhao B, Zeng L, Zhao J, et al. Association of magnesium intake with type 2 diabetes and total stroke: an updated systematic review and meta-analysis. BMJ Open 2020;10:e032240. doi:10.1136/ bmjopen-2019-032240

- Prepublication history and additional material for this paper are available online. To view these files, please visit the journal online (http://dx.doi. org/10.1136/bmjopen-2019032240).

Received 11 June 2019 Revised 09 February 2020 Accepted 12 February 2020

\section{Check for updates}

(c) Author(s) (or their employer(s)) 2020. Re-use permitted under CC BY-NC. No commercial re-use. See rights and permissions. Published by BMJ.

${ }^{1}$ Department of Cardio-Thoracic Surgery, The Second Affiliated Hospital of Nanchang University, Nanchang, Jiangxi, China

${ }^{2}$ Department of Cardiovascular Medicine, The Second Affiliated Hospital of Nanchang University, Nanchang, China

${ }^{3}$ Department of Endocrinology, The Second Affiliated Hospital of Nanchang University, Nanchang, China

${ }^{4}$ Department of Neurology, The Second Affiliated Hospital of Nanchang University, Nanchang, China

Correspondence to Dr Wenxiong Zhang; zwx123dr@126.com

\section{ABSTRACT}

Objective The detailed associations between type 2 diabetes (T2D) and total stroke and magnesium intake as well as the dose-response trend should be updated in a timely manner.

Design Systematic review and meta-analyses.

Data sources PubMed, Embase, Cochrane Library, Web of Science and ClinicalTrials.gov were rigorously searched from inception to 15 March 2019.

Eligibility criteria Prospective cohort studies investigating these two diseases were included.

Data synthesis Relative risk (RR) and $95 \% \mathrm{Cl}$ in random effects models as well as absolute risk (AR) were pooled to calculate the risk of T2D and stroke. Methodological quality was assessed by the Newcastle-Ottawa Scale.

Results Forty-one studies involving 53 cohorts were included. The magnitude of the risk was significantly reduced by $22 \%$ for T2D (RR 0.78 (95\% Cl 0.75 to 0.81 ); $\mathrm{p}<0.001$; AR reduction $0.120 \%), 11 \%$ for total stroke (RR 0.89 (95\% Cl 0.83 to 0.94); $p<0.001$; AR reduction $0.281 \%$ ) and $12 \%$ for ischaemic stroke (RR 0.88 (95\% $\mathrm{Cl} 0.81$ to 0.95$) ; p=0.001$; AR reduction $0.246 \%$ ) when comparing the highest magnesium intake to the lowest. The inverse association still existed when studies on T2D were adjusted for cereal fibre (RR $0.79 ; p<0.001)$ and those on total stroke were adjusted for calcium (RR 0.89; $p=0.040$ ). Subgroup analyses suggested that the risk for total and ischaemic stroke was significantly decreased in females, participants with $\geq 25 \mathrm{mg} / \mathrm{m}^{2}$ body mass index and those with $\geq 12$-year follow-up; the reduced risk in Asians was not as notable as that in North American and European populations.

Conclusions Magnesium intake has significantly inverse associations with T2D and total stroke in a dosedependent manner. Feasible magnesium-rich dietary patterns may be highly beneficial for specific populations and could be highlighted in the primary T2D and total stroke prevention strategies disseminated to the public. PROSPERO registration number CRD42018092690.

\section{INTRODUCTION}

Diabetes is a global burden with an alarming increasing rate throughout the world. ${ }^{12}$ Stroke is an independent disorder and a typical macrovascular complication
Strengths and limitations of this study

In this study, we performed an updated comprehensive quantitative analysis focusing on the dietary effect of magnesium intake.

- The study identified an inverse association between magnesium intake and T2D and stroke.

- A quite number of prospective cohort studies were employed to guarantee the robust evidence.

- Limitation of study was non-inclusion of randomised controlled trails to prove the causality.

- Cases ascertainments are limited by food frequency questionnaires or self-reports.

of type 2 diabetes (T2D), and it is regarded as the second leading cause of death after ischaemic heart disease. ${ }^{34}$ These pandemic health problems necessitate better primary prevention strategies.

Magnesium, a common cellular ion, acts as a critical cofactor for hundreds of enzymes involved in glucose metabolism, protein production and nucleic acid synthesis. ${ }^{56}$ Low levels of magnesium have been associated with many chronic and inflammatory diseases, such as Alzheimer's disease, asthma, attention deficit hyperactivity disorder, insulin resistance, T2D, hypertension, cardiovascular disease (CVD; eg, stroke), migraine headaches, osteoporosis and cancer. ${ }^{1578}$

Notably, many adults in developed countries do not consume the recommended daily amount of magnesium-rich foods such as whole grains, nuts and green leafy vegetables, and magnesium is less mentioned in dietary guidelines and in studies on T2D or stroke prevention. ${ }^{9}{ }^{10}$ Thus, we chose T2D and stroke as our outcome of interest (CVD was not evaluated because there is already a wealth of research relating to CVD, and the definitions of CVD vary greatly among studies, which would increase the heterogeneity in 
the pooled process and impair our interpretation of the final conclusions). Emerging studies ${ }^{11-51}$ on this topic are limited, and the results remain mixed. For example, most studies have indicated that magnesium intake has an inverse association with T2D or total stroke incidence; however, several others have revealed that there is an inverse trend but not a significant association, which is possibly due to limitations related to small sample sizes and differences in the intervention duration, study design and participant characteristics. Moreover, consecutive meta-analyse ${ }^{52}{ }^{53}$ have used less rigorous inclusion; the results were not comprehensive, and they did not completely address the influence of other confounders (ie, body mass index (BMI), cereal fibre, calcium, potassium) on the relationship. Accordingly, we performed a meta-analysis to (1) establish a comprehensive estimate and update the epidemiological evidence for clinical practice; (2) discuss the results of stroke subtype and the impact of several statistical and epidemiology confounders on the investigated association; and (3) highlight the details of the dose-response pattern observed among the participants analysed in the studies.

\section{METHODS}

This study was reported according to the Preferred Reporting Items for Systematic Reviews and Meta-Analyses guidelines (online supplementary table S1) and the Metaanalysis of Observational Studies in Epidemiology Guidelines Checklist (online supplementary table S2).

\section{Search strategy}

PubMed, Embase, Cochrane Library, Web of Science and ClinicalTrials.gov were systematically reviewed through inception to 15 March 2019, for studies on magnesium intake and T2D or stroke without language restrictions. The following key words were used: "Magnesium", "Type 2 Diabetes Mellitus", "Type 2 Diabetes", "Stroke", "Cerebrovascular Stroke", "Cohort Studies" and "Prospective Studies". We also manually searched the reference lists of the retrieved literature (including meta-analyses and brief reports), bibliographies and grey literature (including presentations and unpublished literature) for further eligible articles. The search strategy can be found in online supplementary table S3.

\section{Selection criteria}

(1) Eligible populations must be composed of individuals with plausible dietary/energy intake who had no history of diabetes and/or insulin treatment for T2D analysis and no current stroke for stroke analysis. (2) Their apparent life expectancy was long enough for proper follow-up. (3) We included only prospective cohort studies that reported magnesium intake and T2D and/or various types of stroke. (4) The follow-up duration of eligible studies was at least 1 year if they provided follow-up data. Notably, magnesium intake consisted of both dietary magnesium intake and total magnesium intake (dietary and supplementary magnesium).

Only studies containing the most comprehensive information on the population or endpoints were included to avoid duplication. We excluded reviews, basic science studies, meta-analyses, studies on gestational diabetes mellitus and studies that focused only on magnesium supplementation.

\section{Data extraction and quality assessments}

Two researchers independently extracted the following information: the first author, publication year, period of cohort studies, duration of persistent exposure, basic characteristics of the enrolled participants (weight, age, region, BMI, drinking and smoking habits (previous plus current), etc), median magnesium intake for each quantile (tertile, quartile, or quintile), diabetes and total stroke cases, subtypes of total stroke, dietary and case assessments, adjusted confounding covariates. Importantly, total stroke is classified as clinical ischaemic stroke $(87 \%)$, haemorrhagic stroke $(13 \%)$ and undetermined stroke. ${ }^{54}$ Haemorrhagic stroke is classified as subarachnoid haemorrhage and intracerebral haemorrhage according to anatomical site or presumed aetiology. ${ }^{55}$ In cases of continuing disagreement, a final decision was reached after discussion with a third member of the panel.

Methodological quality was described by the NewcastleOttawa Scale (NOS), which was validated for assessment of the quality of non-randomised controlled trials (RCTs) in meta-analyses. ${ }^{56}$ For the $0-10$ scale, each study was categorised as low (0-5), medium (6-7) or high (8-10) quality.

\section{Statistical analysis}

Articles providing data separately for men and women or black and white or different types of disease within an article were treated as independent studies. Multivariate relative risk (RR) and corresponding 95\% CI as well as absolute risk (AR) for measuring the quantitative associations between exposure and T2D, total stroke and other wanted outcomes, particularly for the highest versus the lowest categories of magnesium intake, were estimated by the DerSimonian-Laird random effects model because the assumptions involved account for the presence of within-study and between-study variability. Statistical heterogeneity was determined with the Cochran $Q \chi^{2}$ test and the $I^{2}$. An $I^{2}>50 \%$ or a $\mathrm{p}$ value for the $\mathrm{Q}$ test $<0.1$ was considered to indicate significant heterogeneity. ${ }^{57}$ We performed sensitivity analyses to test the robustness and postsubgroup analyses to detect the source of heterogeneity. In addition, a random effects meta-regression analysis on BMI, sex, participant region and dietary assessments with RR for each trial was performed to obtain an understanding of the reasons for heterogeneity. RR and 95\% CI might begin to significantly change as publication years increased in T2D and total stroke, which would be validated by cumulative meta-analyses. 
The dose-response analyses for all outcomes were proposed by Greenland and Longnecker ${ }^{58}$ and Orsini $e t$ $a p^{59}$. The categories of magnesium intake, distributions of cases and person year, RR and 95 CI were extracted. If the number of cases and/or person years was not available, variance-weighted least squares regression was used to pool the risk estimate. For most studies, the median intake for each quantile (tertile, quartile or quintile) of magnesium intake was assigned as the representative dose. For continuous intake, which was reported as categorical data (range) in some studies, we assigned the midpoint category of the lower and upper bounds to the RR in these studies; when the highest category was open ended, we assumed the length of the open-ended interval to be 1.5 times the adjacent interval; when the lowest category was open, we assigned the adjacent interval of the category to be 1.5 times the length of the open-ended interval. We employed generalised least squares regression models to calculate study-specific RR estimates per $50 \mathrm{mg} /$ day, $100 \mathrm{mg} /$ day and $150 \mathrm{mg} /$ day magnesium intake increment if there was evidence of a linear relationship. Nonlinear relationships between magnesium intake and all outcomes were evaluated using restricted cubic splines with four knots located at the 5th, 35th, 65th and 95th percentiles of the distribution. The $p$ value for curve linearity or nonlinearity was calculated by testing the null hypothesis that the coefficient of the second spline is equal to zero. All results were presented using two-stage dose-response model plots (including linear and nonlinear relationships). Some results were demonstrated as forest plots for intake increments of $<50 \mathrm{mg}$ / day, $\geq 50$ and $<100 \mathrm{mg} /$ day, $\geq 100$ and $<150 \mathrm{mg} /$ day, and $\geq 150 \mathrm{mg} /$ day.

Publication bias was assessed graphically by Begg's adjusted rank correlation funnel plots ${ }^{60}$ and Egger's linear regression tests. ${ }^{61}$ All analyses were performed using Stata V.14.0 (StataCorp); two-sided $\mathrm{p}<0.05$ was considered statistically significant except where otherwise specified.

\section{Patient and public involvement}

No patients were involved in developing the research question or the outcome measures, and no patients were involved in planning the design or implementation of the study. Furthermore, no patients were asked to advise on the interpretation or write-up of the results. Since this study used aggregated data from previous publications, it is not easy to disseminate the results of the research to study participants directly.

\section{RESULTS}

\section{Study characteristics and quality assessment}

Of the 8713 studies, 107 studies were considered for eligibility after screening the titles and abstracts (figure 1). A total of $41^{11-51}$ prospective cohort studies comprising 53 cohorts, 1912634 participants and 76678 cases were eligible for inclusion in the systematic review and metaanalysis (online supplementary table S4). Hodge $e a^{18}$ recorded only $500 \mathrm{mg}$ /day increments of magnesium for further pooled analyses; two studies ${ }^{3351}$ failed to clearly distinguish the diabetes type, but the vast majority of cases had T2D. We computed the subtype data in three studies ${ }^{1427} 36$ after the extraction of total stroke, and we regarded ischaemic stroke in three other studies ${ }^{28} 3042$ as total stroke given that ischaemic stroke accounted for nearly $87 \%$ of total stroke. Participants were predominately middle aged at baseline, with a mean magnesium intake of $370 \mathrm{mg} /$ day for the highest category and $232 \mathrm{mg}$ /day for the lowest category. The mean duration of all eligible studies was 10.7 years. Nineteen studies were conducted in North America (America); 5 studies were conducted in Europe (Sweden, the Netherlands and Britain); 13 studies were conducted in Asia (China and Japan and Taipei); and 4 studies enrolled individuals in multiple nations. Most of the included studies used food frequency questionnaires (FFQs) or semiquantitative FFQs to assess individual dietary intake. Eighteen studies used dietary magnesium intake, and 21 studies recorded total magnesium intake (dietary and supplementary magnesium intake). Of note, supplementary magnesium intake was assessed by the use of magnesium or multivitamin supplements; nevertheless, dietary magnesium accounted for the majority of magnesium intake. Adjusted confounders were mostly similar; however, adjusted dietary confounders such as cereal fibre, potassium and calcium still varied across individual studies. It was unclear whether the included studies had adjusted for sodium because they did not provide this information. All the studies were written in English.

After the quality assessments of the studies according to NOS, the average score was 8.85 (online supplementary table S5), and all studies were of high quality (NOS scores 8-10).

\section{Magnesium intake and T2D incidence}

Thirty-five cohorts from 26 publications ${ }^{11} 121520$ 22-26 2931-35 37394143484951 (1 219636 participants and 56540 T2D cases) reported that the magnitude of T2D risk was reduced by 22\% (RR 0.78 (95\% CI 0.75 to 0.81$) ; \mathrm{p}<0.001$; AR reduction $0.120 \%$ ), comparing the highest category of magnesium intake to the lowest, with little evidence of heterogeneity $\left(I^{2}=35.6 \%\right.$; $\left.\mathrm{p}=0.021\right)$. The dose category-specific analysis suggested that for the $<50 \mathrm{mg}$ /day magnesium increment, the risk of T2D was reduced by $10 \%$ (RR 0.90 (95\% CI 0.88 to 0.93 ); $\mathrm{p}<0.001)$; for the $\geq 50$ and $<100 \mathrm{mg} /$ day increments, the risk was decreased by $16 \%$ (RR 0.84 (95\% CI 0.82 to $0.87) ; \mathrm{p}<0.001)$; for $\geq 100$ and $<150 \mathrm{mg} /$ day increments, the risk was reduced by $22 \%$ (RR 0.78 (95\% CI 0.74 to $0.83)$; $\mathrm{p}<0.001$ ); and for the $\geq 150 \mathrm{mg}$ /day increment, the risk was reduced by $21 \%$ (RR 0.79 (95\% CI 0.74 to 0.84 ); $\mathrm{p}<0.001$; figure 2). Little evidence of publication bias was found (Egger's test: $\mathrm{p}=0.088$; online supplementary figure S1A). 


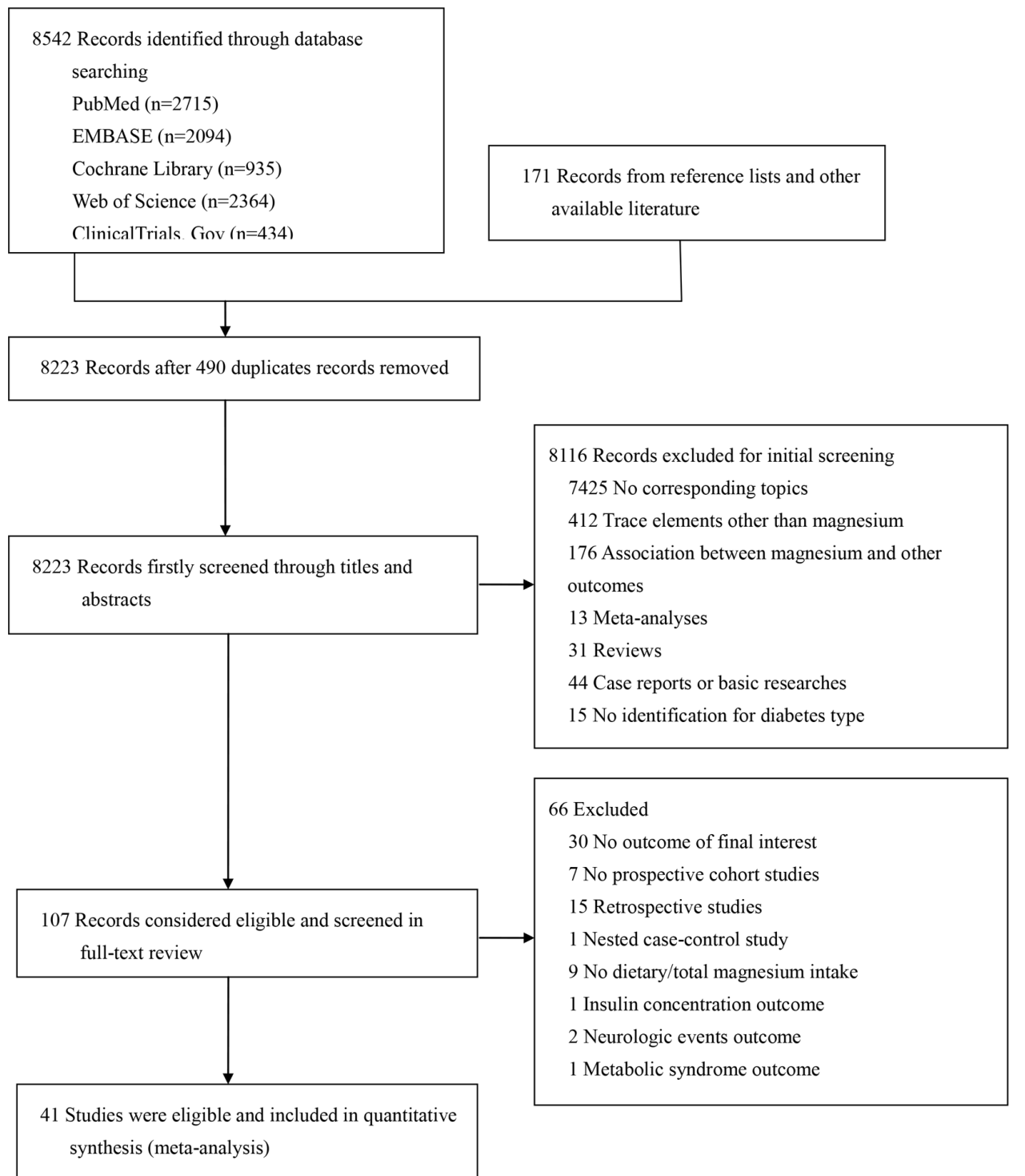

Figure 1 Flow chart for the literature search and screening process.

\section{Magnesium intake and stroke incidence}

Eighteen cohorts from 15 publica-

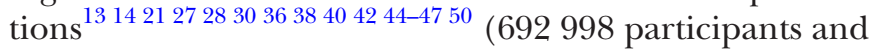
20138 total stroke cases) reported that the magnitude of the risk of total stroke was decreased by $11 \%$ (RR 0.89 (95\% CI 0.83 to 0.94$)$; $\mathrm{p}<0.001$; AR reduction $0.281 \%$ ), comparing the highest category of magnesium intake with the lowest, with no heterogeneity $\left(I^{2}=0 \% ; \mathrm{p}=0.529\right)$. The dose category-specific analysis revealed no significant association with the $<50 \mathrm{mg} /$ day, $\geq 50$ and $<100 \mathrm{mg} /$ day increments or the $\geq 100$ and $<150 \mathrm{mg}$ /day increments. For the $\geq 150 \mathrm{mg}$ / day increment, the risk of total stroke was decreased by $15 \%$ (RR 0.85 (95\% CI 0.79 to 0.91 ); $\mathrm{p}<0.001$; online supplementary figure S2). Publication bias was evaluated for stroke subtypes.

Fifteen cohorts from 12publications ${ }^{142127283036384042454650}$ reported ischaemic stroke. The magnitude of the risk of ischaemic stroke was reduced by $12 \%$ (RR 0.88 (95\% CI 0.81 to 0.95$) ; \mathrm{p}=0.001$; AR reduction $0.246 \%$ ) with no significant heterogeneity $\left(I^{2}=16.9 \% ; \mathrm{p}=0.265\right)$. The dose category-specific analysis identified no significant association with the $<50 \mathrm{mg} /$ day, $\geq 50$ and $<100 \mathrm{mg} /$ day, or $\geq 100$ and $<150 \mathrm{mg} /$ day increments. A decreasing trend existed but remained non-significant. The original risk was reduced by $16 \%$ in the analysis of the $\geq 150 \mathrm{mg}$ / day increment (RR 0.84 (95\% CI 0.78 to 0.91$)$; p<0.001; online supplementary figure S3). No publication bias was observed in terms of ischaemic stroke (Egger's test: $\mathrm{p}=0.937$; online supplementary figure S1B).

Ten cohorts from eight studies ${ }^{1421273638454650}$ reported that haemorrhagic stroke was not significantly associated with magnesium intake (RR 0.93 (95\% CI 0.82 to 1.06 ); $\mathrm{p}=0.282)$. The dose category-specific analysis identified no significant association (online supplementary figure S4). No significant heterogeneity or publication bias was observed in terms of haemorrhagic stroke (Egger's test: $\mathrm{p}=0.809$; online supplementary figure $\mathrm{S} 1 \mathrm{C}$ ) .

Three publications involving three cohorts ${ }^{14} \quad 27 \quad 36$ showed that high magnesium intake had no significant effect on reducing the risk of subarachnoid haemorrhage 


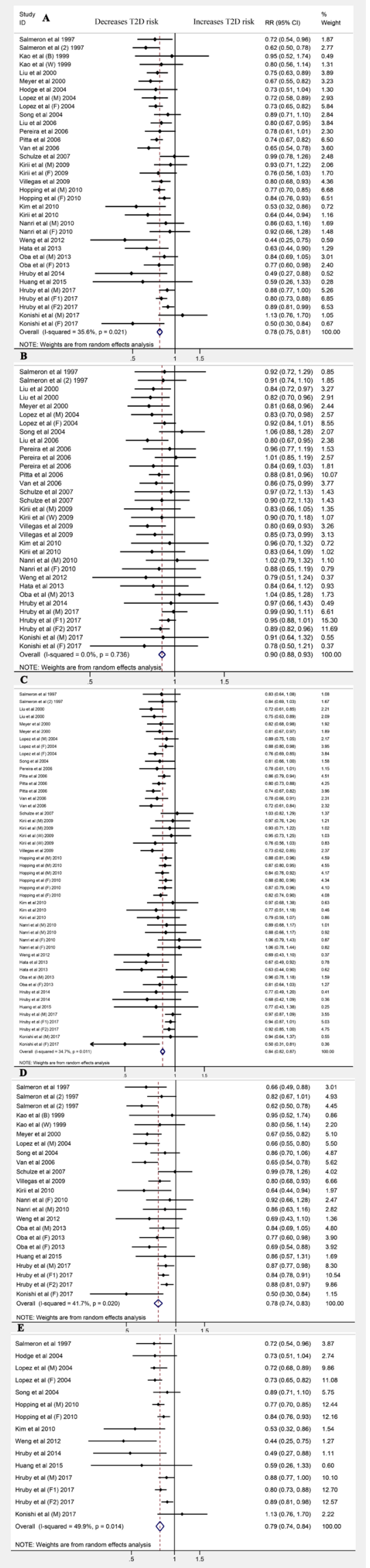

Figure 2 Forest plots for the risk of type 2 diabetes (T2D) for (A) magnesium intake and for (B) $<50 \mathrm{mg} /$ day, (C) $\geq 50$ and $<100 \mathrm{mg} /$ day, $(D) \geq 100$ and $<150 \mathrm{mg} /$ day and $(E) \geq 150 \mathrm{mg} /$ day increments $(E)$. 
(RR 0.99 (95\% CI 0.71 to 1.39 ); $\mathrm{p}=0.963$ ). The dose category-specific analysis revealed no significant association (online supplementary figure S5).

With respect to intracerebral haemorrhage, the pooled results from three cohorts ${ }^{142736}$ in three publications revealed no significant advantages of intracerebral haemorrhage (RR $0.92(95 \%$ CI 0.71 to 1.20$) ; \mathrm{p}=0.540)$. The dose category-specific analysis revealed no significant association (online supplementary figure S6).

\section{Meta-regression and cumulative meta-analysis}

According to the meta-regression results, there was no evidence of BMI, sex, participant region or dietary assessment for each individual trial bias in terms of T2D (online supplementary figure S7), total stroke (online supplementary figure S8), ischaemic stroke (online supplementary figure S9) and haemorrhagic stroke events (online supplementary figure $\mathrm{S} 10)$. The male subgroup ( $\mathrm{p}=0.041$ ) in the sex category might lead to slight heterogeneity in terms of total stroke; however, sex $(p=0.112)$ showed no association with total stroke incidence.

Analyses of T2D (online supplementary figure S11), total stroke (online supplementary figure S12) and ischaemic stroke demonstrated that the RRs of the final results became robust within a narrow range and remained significant as publication years increased and more recent high-quality studies were included. After inclusion of the Iso $e a^{14}$ study, the RR and 95\% CI for ischaemic stroke decreased to less than 1 and then became stable (online supplementary figure S13). Although there was no significant reduction in the risk of haemorrhagic stroke, evidence clearly showed that the CI was becoming narrow, which trended towards significance (online supplementary figure S14). Thus, the risk for haemorrhagic stroke might be reduced; additional studies are warranted.

\section{Sensitivity analysis}

When three ${ }^{24-26}$ studies were excluded from the T2D analysis, the summary RR changed from 0.78 (95\% CI 0.75 to 0.81 ) to 0.78 (95\% CI 0.75 to 0.82 ), with the heterogeneity declining from $\left(I^{2}=35.6 \% ; \mathrm{p}=0.021\right)$ to $\left(I^{2}=24.0 \% ; \mathrm{p}=0.112\right)$. Among T2D analyses, eight studies $^{19} 22232633394849$ adjusted for cereal fibre intake yielded an RR of 0.79 (95\% CI 0.73 to 0.85 ; $\mathrm{p}<0.001$ ), and two studies ${ }^{1535}$ adjusted for calcium yielded an RR of 0.87 ( $95 \%$ CI 0.73 to $1.04 ; \mathrm{p}=0.128$ ). Among the total stroke analysis, the summary RR was 0.92 (95\% CI 0.82 to 1.02 ; $\mathrm{p}=0.097$ ) in five studies ${ }^{13} 44-4650$ adjusted for potassium intake and was 0.89 (95\% CI 0.80 to $0.99 ; \mathrm{p}=0.040$ ) in five studies $^{1444-46} 50$ adjusted for calcium. Only one study ${ }^{15}$ adjusted for potassium intake in T2D, and one study ${ }^{36}$ adjusted for cereal fibre in total stroke.

\section{Subgroup analysis}

Stratified analyses by characteristics of the population and study design were conducted on T2D (table 1), total stroke, ischaemic stroke and haemorrhagic stroke (table 2). The inverse association with T2D remained robust across all subgroups with little evidence of heterogeneity. For stroke incidence, a decreased risk of total stroke and ischaemic stroke was found in female participants (RR 0.91 (95\% CI 0.83 to 0.99) for total stroke; 0.89 (95\% CI 0.79 to 1.00 ) for ischaemic stroke) and individuals with $\geq 25 \mathrm{~kg} / \mathrm{m}^{2}$ mean BMI (RR 0.89 (95\% CI 0.82 to 0.96 ) for total stroke; 0.88 (95\% CI 0.81 to 0.96 ) for ischaemic stroke). When restricted to $\mathrm{a} \geq 12$-year follow-up, the risk of total stroke and ischaemic stroke was significantly reduced (RR 0.89 (95\% CI 0.83 to 0.95 ) for total stroke; 0.88 (95\% CI 0.81 to 0.95 ) for ischaemic stroke). These risks were more reduced in North American and European individuals than in Asians. Cardiovascular events (CV events, coronary heart disease, heart failure, atrial fibrillation, self-reported heart disease, etc, other than stroke), hypercholesterolaemia and diabetes would blunt the effect of magnesium on total and ischaemic stroke. However, magnesium intake could still, or at least, demonstrate the trend to decrease total and ischaemic stroke in individuals even with those risk factors. Similarly, CV events, hypercholesterolaemia and family diabetes history had no substantial impact on the inverse association between T2D incidence and magnesium intake. We did not find a significantly reduced risk of haemorrhagic stroke in the subgroup analyses.

\section{Dose-response analysis}

In this part, both linear and nonlinear relationships were found in T2D (figure 3A), in total stroke (figure 3B) and in ischaemic stroke (figure 3C). However, no linear or nonlinear dose-response relationship was observed in haemorrhagic stroke (figure 3D) along with the subtypes including subarachnoid haemorrhage and intracerebral haemorrhage (online supplementary figure S15).

Specifically, we calculated the RR for the magnesium increments if a linear relationship was found. The calculated RR was 0.94 (95\% CI 0.93 to 0.95 ) for the $100 \mathrm{mg}$ / day increment for T2D. For total stroke, the summary RR was 0.98 (95\% CI 0.97 to 0.99 ) related to a $100 \mathrm{mg} /$ day increment in magnesium intake, and the RR for ischaemic stroke was 0.98 (95\% CI 0.97 to 0.99 ) related to a $100 \mathrm{mg} /$ day increment in magnesium intake. There was no RR cut-off point at which the decreasing trend reversed, but the RR decreased slightly rapidly with any slight decreases at approximately $260 \mathrm{mg}$ /day for T2D and $350 \mathrm{mg}$ /day for total/ischaemic stroke. However, there was substantial uncertainty in the lower range of this distribution (figure 3A-C).

\section{DISCUSSION \\ Main findings}

This paper used a general and up-to-date search strategy to identify additional studies that were missed in prior meta-analyses under real-world conditions. Our results support a significant inverse association between magnesium consumption and T2D, total stroke and ischaemic stroke at the highest level versus the lowest. No significant 
Table 1 Subgroup analysis relating to magnesium intake and type 2 diabetes (T2D)

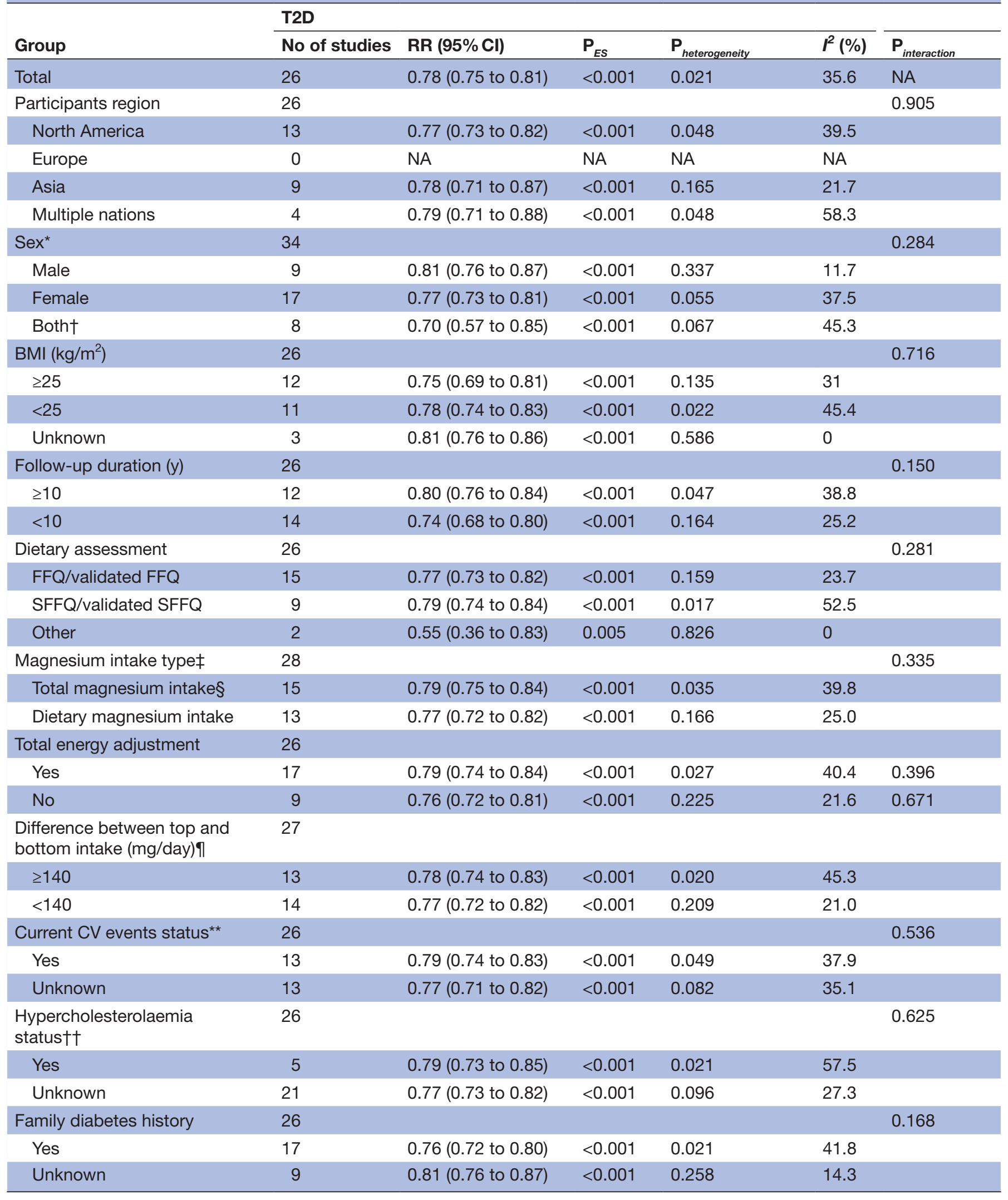

Continued 


\begin{tabular}{|c|c|c|c|c|c|c|}
\hline \multirow[b]{2}{*}{ Group } & \multicolumn{5}{|l|}{ T2D } & \\
\hline & No of studies & RR $(95 \%$ Cl) & $\mathbf{P}_{E S}$ & $\mathbf{P}_{\text {heterogeneity }}$ & $I^{2}(\%)$ & $\mathbf{P}_{i n t e}$ \\
\hline
\end{tabular}

*Male and female of T2D outcome were treated as independent cohorts within eight studies.

†Male and female participants were in independent cohorts.

$\ddagger$ Two studies reported total magnesium and dietary magnesium intake outcome.

$\S$ Total magnesium intake ( $\mathrm{mg} / \mathrm{day}$ ) included the total amount of magnesium from both food (diet) and supplement.

IS Subtract the lowest category intake from the highest. Oba el al (M) was in $<140$ group, while Oba el al (F) was in $\geq 140$ group.

${ }^{* *}$ Grouped by whether participants with or without cardiovascular events (CV events). CV events in this part include coronary heart disease, heart failure, stroke, atrial fibrillation and self-reported heart disease.

$\dagger+G$ rouped by whether participants with or without hypercholesterolaemia. Hypercholesterolaemia in this part means cholesterol concentration $\geq 240 \mathrm{mg} / \mathrm{dL}$.

BMI, body mass index; ES, effect size; FFQ, food frequency questionnaire; NA, not available; RR, relative risk; SFFQ, semiquantitative food frequent questionnaire.

association for haemorrhagic stroke, subarachnoid haemorrhage or intracerebral haemorrhage was detected. Female participants with obesity (mean BMI $\geq 25 \mathrm{~kg} / \mathrm{m}^{2}$ ) with a longer follow-up period ( $\geq 12$ years) might obtain greater benefit from magnesium intake with a lower risk of total and ischaemic stroke incidence. In subgroup analyses, the RR of stroke risk was highly decreased among North American and European individuals. Significant risk was reduced by $6 \%, 2 \%$ and $2 \%$ for T2D, total stroke and ischaemic stroke, respectively, per $100 \mathrm{mg}$ /day increment in magnesium intake level. Overall, our study supports the guidelines to address the role of magnesium intake in early prevention strategies to combat T2D and stroke. However, additional RCTs are needed in the future to validate the causality.

\section{Clinical implications}

Dietary nutrients are popular topics for current clinical medicine; folic acid, vitamin $\mathrm{D}$ and $\omega-3$ fatty acids have been specifically recommended to pregnant women, infants and children, and the elderly ${ }^{62}{ }^{63}$ However, magnesium has been less extensively discussed. This is a noteworthy study for the following reasons. First, the current study reinforces the possible role of magnesium in the prevention and management of two chronic illnesses and invites new considerations regarding the potential avoidance of other chronic diseases through dietary strategies. Second, this comprehensive study including nearly two million individuals and possessing abundant statistical power provides confirmatory evidence for medical practitioners, health educators and policy-makers. Third, to date, no related paper has discussed such detailed stratified analyses; thus, this work helps physicians amplify dietary benefits through individualised strategies. Interestingly, North American and European participants seemed to receive more benefits from magnesium intake than Asians. Fourth, to the best of our knowledge, this is the first study in which a cumulative meta-analysis was performed to predict changes in the tendency of main risk estimates. Based on past and current cutting edge evidence about nutrition and T2D prevention, the US Diabetes Prevention Program conducted a study and demonstrated that proper lifestyle modification (exercise and Mediterranean diet) significantly reduced T2D risk irrespective of population baselines, and this benefit was enhanced with increased follow-up. ${ }^{64}$ The UK National Health Service will launch an intervention programme including weight loss, nutrition, monitoring and peer support targeting up to 10000 people prone to develop T2D. ${ }^{65}$

The 2018 American Diabetes Association guidelines ${ }^{66}$ recommend that the intake of nuts, berries, yoghurt, coffee and tea be increased in individuals who are at high risk of diabetes. The latest guidelines by the American Heart Association/American Stroke Association ${ }^{9}$ also validate the considerable status of early management of stroke (ischaemic stroke). In fact, magnesium is a cofactor in enzyme systems that regulate diverse biomedical reactions, including protein synthesis, muscle and nerve transmission, neuromuscular conduction, signal transduction blood glucose control and blood pressure (BP) management. ${ }^{67}$ Magnesium also plays a role in transporting calcium and potassium ions across the cell membrane and is crucial for the structural function of proteins, nucleic acids or mitochondria. ${ }^{68}$ In diabetes, magnesium is involved in glucose and insulin metabolism by regulating the tyrosine kinase activity of the insulin receptor. Magnesium also influences phosphorylase B kinase activity by releasing glucose-1-phosphate from glycogen and regulates glucose translocation into the cell. ${ }^{69}$ In stroke, higher magnesium levels lead to the deregulation of glutamate and calcium cation influx by reducing N-Methyl-D-aspartic acid (NMDA) receptor activity and blocking voltage-gated calcium channels, eliminating calcium cation cytotoxicity. Additionally, the vasodilatory effects of magnesium may benefit patients who had ischaemic stroke. ${ }^{70}$ Indeed, a poor outcome of haemorrhagic stroke was observed in an RCT; however, high serum magnesium might be better for the prognosis of intracerebral haemorrhage. ${ }^{71}$

Most specific nutrients, especially macronutrients, are correlated with total energy intake. In the included freeliving human studies, the variation in total energy intake originated from differences in physical activity levels, body size and energy efficiency. ${ }^{72}$ Thus, total energy intake can 


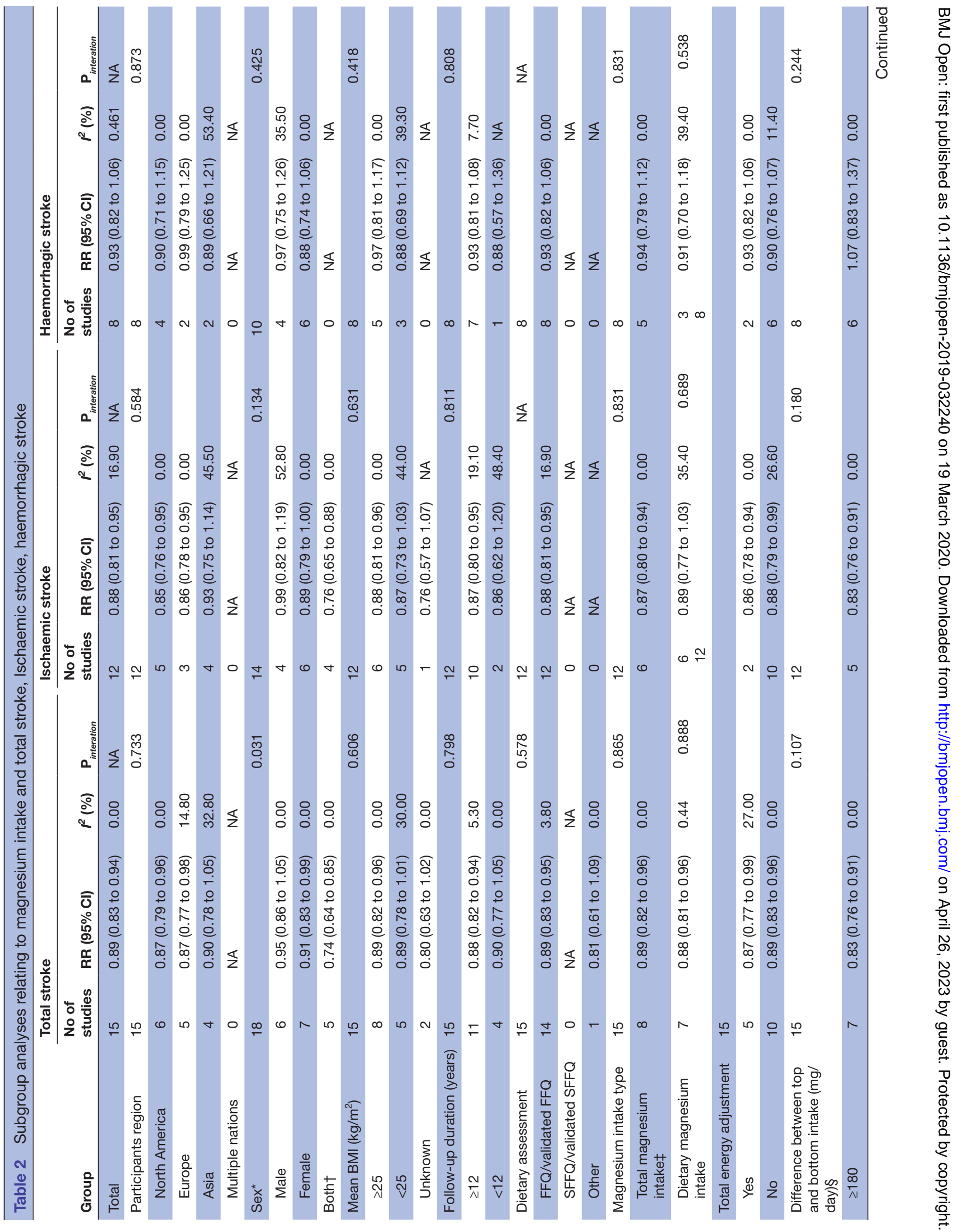




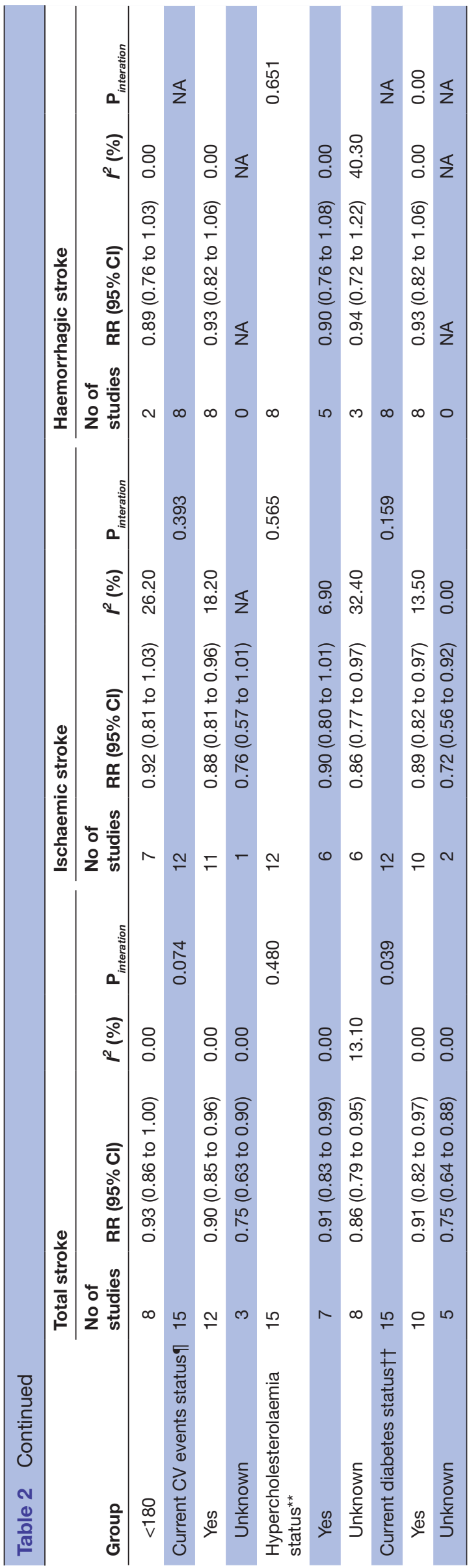

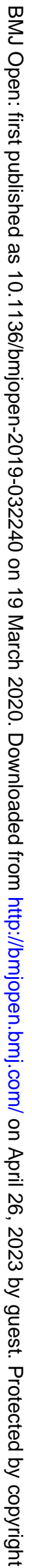




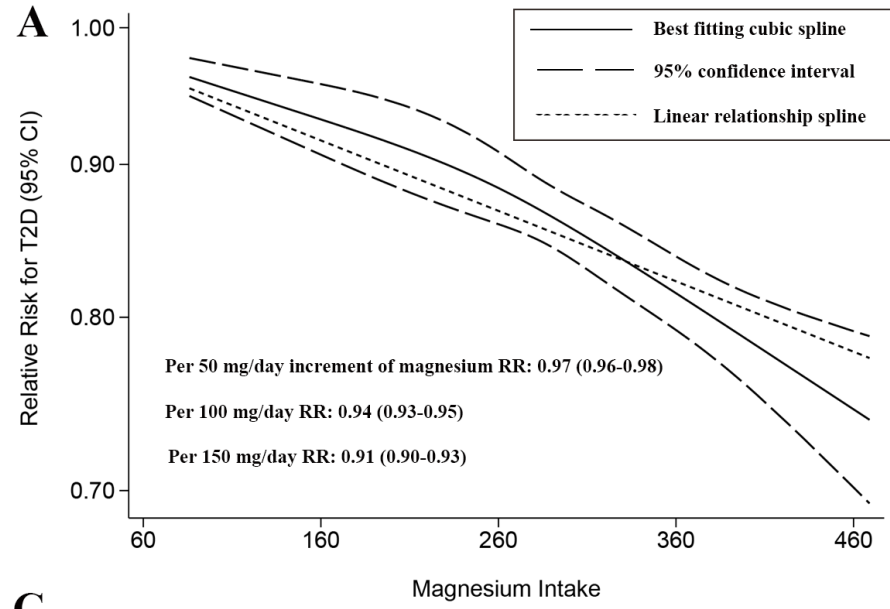

C

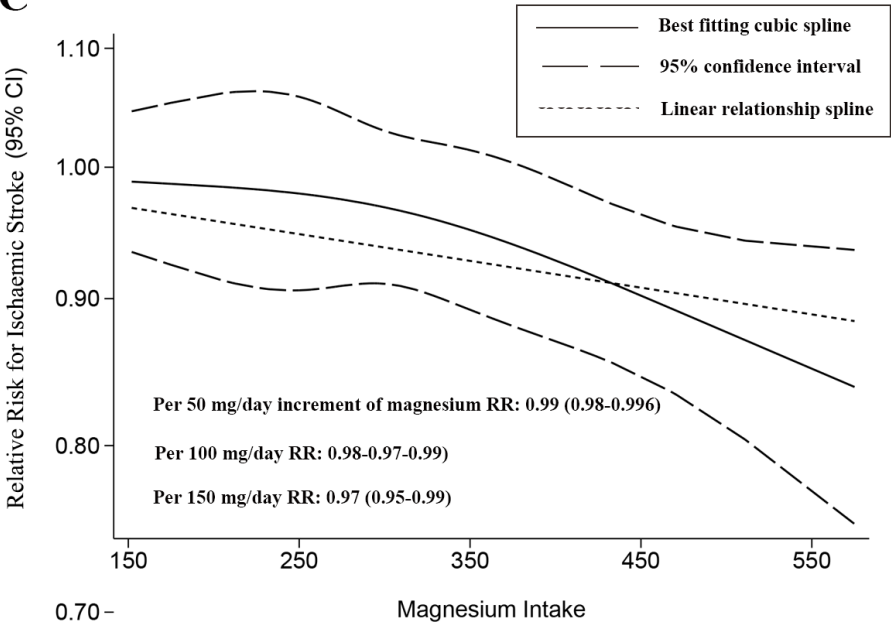

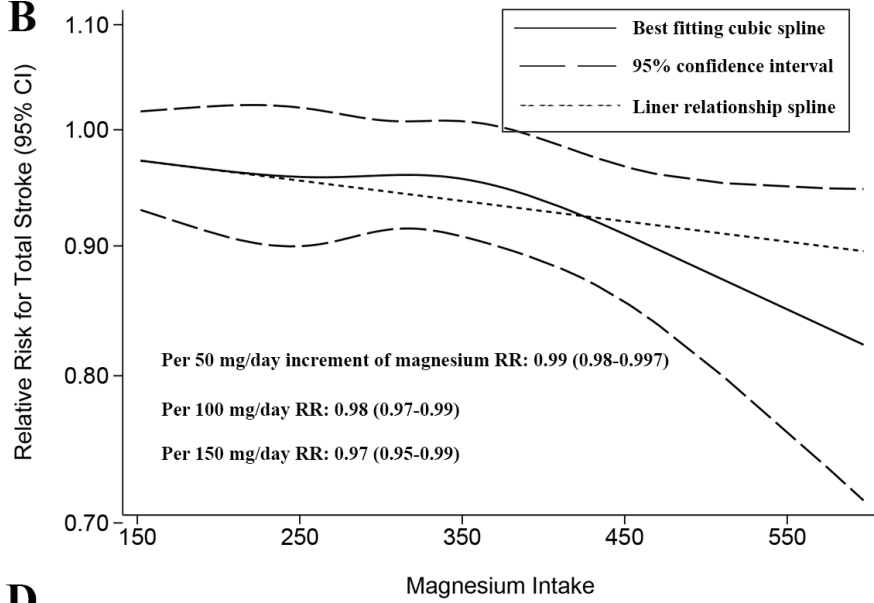

D

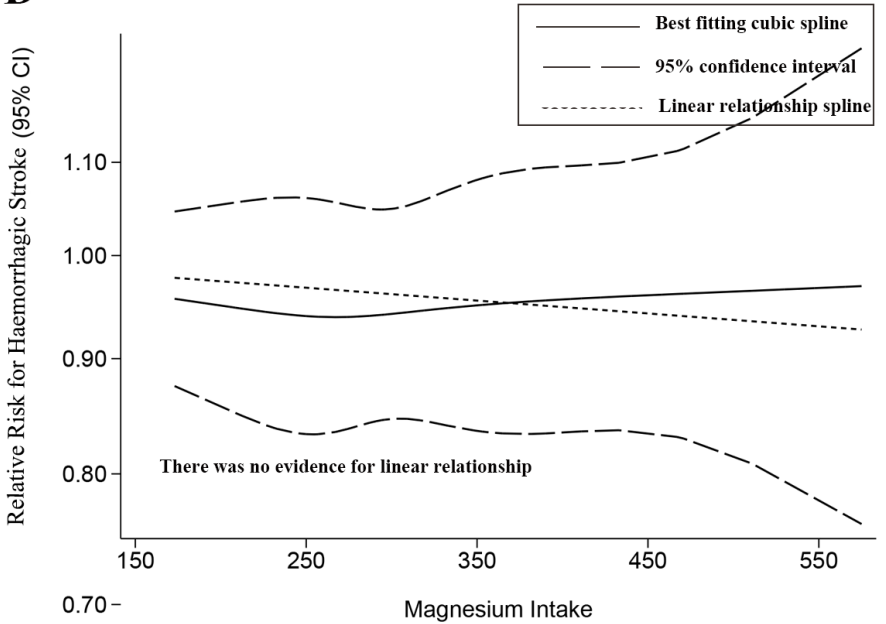

Figure 3 Two-stage dose-response effect on the relationships between magnesium intake and (A) type 2 diabetes (T2D), (B) total stroke, $(C)$ ischaemic stroke and $(D)$ haemorrhagic stroke. RR, relative risk.

weaken the investigated association with considerable nutrient intake if this covariable is not properly removed. Epidemiologists should assess the reproducibility and validity of energy-adjusted nutrients as well as absolute nutrient intake. For micronutrients such as magnesium, an inverse association with T2D, total stroke and ischaemic stroke outcomes could be still found after total energy intake adjustment. In terms of other nutrients, potassium intake is proposed to lower BP and improve vascular outcomes (including stroke); dietary potassium may also be influential in glucose control and limiting the risk of diabetes. ${ }^{73}$ Vitamin D and calcium may negatively influence glycaemia, but evidence is limited and mostly based on cross-sectional observational studies. ${ }^{74}$ Calcium may be inversely associated with stroke in populations with low-to-moderate calcium intakes, but no significant association was found between calcium and CVD. ${ }^{75}$ Altogether, the results indicate that magnesium-rich food such as nuts $(151-567 \mathrm{mg} / 100 \mathrm{~g}$ edibles), fruits (132$448 \mathrm{mg} / 100 \mathrm{~g}$ edibles), vegetables $(132-1257 \mathrm{mg} / 100 \mathrm{~g}$ edibles), legumes (138-243 mg/100 g edibles), fish (143$303 \mathrm{mg} / 100 \mathrm{~g}$ edibles) and total grain (134-306 mg/100 g edibles) should be recommended to populations with insufficient magnesium intake.

\section{Comparisons with other similar studies}

This analysis has several differences from previous studies. Dong et $a \tilde{l}^{2}$ found that magnesium intake had an inverse association with T2D incidence (RR 0.78 (95\% CI 0.73 to $0.84)$ ), and with an intake of $100 \mathrm{mg} /$ day magnesium, the risk was reduced by $14 \%$. However, they failed to include adequate studies, and standard quality assessments of eligible studies were absent. Individuals from multiple nations were included in some studies ${ }^{18} 252632$ but were incorrectly assigned to Asia or the USA in the subgroups; other minor issues also existed in the selection criteria, making it unclear whether they excluded participants with subclinical diabetes. BMI was not a potential modifier for T2D in our study due to the inclusion of more evidence with a longer follow-up period. Fang $e t a l^{76}$ revealed that dietary magnesium was significantly associated with a reduced risk of T2D (RR 0.74 (95\% CI 0.69 to 0.80$)$ ) and stroke (RR 0.88 (95\% CI 0.82 to 0.95$)$ ). The results were comparable, but they focused only on dietary magnesium intake rather than overall magnesium intake (total or dietary), and subtypes of total stroke were missing. To the best of our knowledge, BMI, follow-up, family diabetes history, and so on, are crucial confounders for evaluating the association, and these factors were not addressed 
in their study. Moreover, other researchers have better investigated the likelihood of a linear association in the dose-response pattern (using methods by Greenland and Orsini et $a l)$. For example, Fang $e t a l^{77}$ found that the $100 \mathrm{mg} /$ day intake of dietary magnesium was associated with an $8 \%-13 \%$ reduction in T2D risk, and while a nonlinear relationship did not exist, a minor publication bias was present. Twenty-five studies were eligible; however, some of them focused not on dietary intake but rather on total magnesium intake. Moreover, there were two included studies focusing on red meat intake instead of magnesium intake. After excluding ineligible studies, we found no evidence of publication bias. Additionally, both linear and nonlinear relationships existed for T2D because the RRs of the highest category of magnesium intake versus the lowest in our pooled study were still used. A study by Larsson $e t a \tilde{l}^{3}$ including seven studies supported a modest but statistically significant inverse association between dietary magnesium intake and stroke. However, the sample size was quite small, and there was no useful information on stroke subtypes (eg, ischaemic stroke, haemorrhagic stroke) in the main analysis. In our opinion, a well-designed subgroup analysis is compulsory, and a pooled stroke result restricted by potassium and calcium adjustment is recommended. The current study found that magnesium intake was strongly inversely associated with total stroke and ischaemic stroke, which still existed in the dose-response pattern.

\section{Directions for future research}

Future studies are needed to address some remaining questions. At first, no significant association was found for haemorrhagic stroke; however, a beneficial trend was observed in the cumulative meta-analysis, which highlights the need for more updated prospective studies and RCTs. Second, there is a key question regarding the optimal time to start prevention and methods to screen severe complications. CV events occur in more than $50 \%$ of patients with diabetes, and diabetic kidney disease occurs in 20\%-40\%. Additionally, CV events increase the risk of death threefold to fourfold compared with patients without such complications. A sustained period of intensive glucose control early in T2D has been confirmed to reduce complication rates. ${ }^{78}$ Most importantly, for the public, educators and policy-makers, promoting magnesium-rich food consumption can translate into considerable benefit in preventing T2D and total stroke, especially for high-risk populations.

\section{Limitations}

This work has several limitations that deserve further discussion. First, this group-level meta-analysis is insufficient. Although strong inverse associations for T2D and total stroke were reported, individual-level studies having more detection power are required. Second, several variations cannot be totally understood; for example, we cannot exclude the possibility that other nutrients and/or dietary components correlated with dietary magnesium may have been responsible, either partially or entirely, for the observed associations. Based on eligible studies, we could not quantify the impact of supplementary magnesium (not combined with dietary intake) on T2D and stroke incidence. The real effect of some dietary supplements on T2D or CVD has proven very interesting to a number of medical experts, clinicians and nutrition educators. Third, FFQs/validated FFQs mostly used in primary studies could not characterise all the nutrients, which misclarified plausible associations. It was suggested that magnesium-specific food questionnaires and/or food records should be reasonably used for accurate magnesium intake estimation. Finally, additional RCT are needed, as observational studies might only reach one conclusion (ie, magnesium intake is inversely associated with T2D incidence) and cannot prove causality.

\section{CONCLUSION}

Magnesium intake has a substantial inverse association with T2D and total stroke. Among these populations, magnesium consumption can be recommended as an optimisation for T2D, total stroke and ischaemic stroke primary prevention or early management. In particular, the greater the magnesium intake, the greater the reduction in risk. As patients, physicians, policy-makers and legislators debate these issues, such a cost-effective alternative is needed to inform policy decisions and aid in reforming nutritional healthcare worldwide.

Acknowledgements The authors thank Yanhua Tang, MD (The Second Affiliated Hospital of Nanchang University) for her advice and Xiaoshu Cheng, MD, PhD (The Second Affiliated Hospital of Nanchang University) for his data collection.

Contributors BZ had full access to all of the data in the manuscript and takes responsibility for the integrity of the data and the accuracy of the data analysis. Concept and design: all authors. Acquisition, analysis or interpretation of data: all authors. Drafting of the manuscript: BZ and WZ. Critical revision of the manuscript for important intellectual content: BZ, LZ, JZ, QW, FZ, LG and YD. Statistical analysis: BZ. Supervision: WZ and YW.

Funding This study was supported by National Natural Science Foundation of China (NSFC), number of grants (81560345), Natural Science Foundation of Jiangxi Province (Grant number: 20161BAB215237).

Competing interests None declared.

Patient consent for publication Not required.

Provenance and peer review Not commissioned; externally peer reviewed.

Data availability statement All data relevant to the study are included in the article or uploaded as supplementary information.

Open access This is an open access article distributed in accordance with the Creative Commons Attribution Non Commercial (CC BY-NC 4.0) license, which permits others to distribute, remix, adapt, build upon this work non-commercially, and license their derivative works on different terms, provided the original work is properly cited, appropriate credit is given, any changes made indicated, and the use is non-commercial. See: http://creativecommons.org/licenses/by-nc/4.0/.

ORCID iD

Wenxiong Zhang http://orcid.org/0000-0003-2962-0847

\section{REFERENCES}

1 Centers for Disease Control and Prevention. National diabetes statistics report, 2017. Atlanta, GA: Centers for Disease Control 
and Prevention, US Department of Health and Human Services, 2017.

2 Zhou B, Lu Y, Hajifathalian K, et al. Worldwide trends in diabetes since 1980: a pooled analysis of 751 population-based studies with 4.4 million participants. Lancet 2016;387:1513-30.

3 Benjamin EJ, Blaha MJ, Chiuve SE, et al. Heart disease and stroke Statistics-2017 update: a report from the American heart association. Circulation 2017;135:e146-603.

4 Feigin VL, Forouzanfar MH, Krishnamurthi R, et al. Global and regional burden of stroke during 1990-2010: findings from the global burden of disease study 2010. Lancet 2014;383:245-55.

5 Barbagallo M, Dominguez LJ. Magnesium metabolism in type 2 diabetes mellitus, metabolic syndrome and insulin resistance. Arch Biochem Biophys 2007;458:40-7.

6 Zhao L, Zhang F, Ding X, et al. Gut bacteria selectively promoted by dietary fibers alleviate type 2 diabetes. Science 2018;359:1151-6.

7 Reffelmann T, Ittermann T, Dörr M, et al. Low serum magnesium concentrations predict cardiovascular and all-cause mortality. Atherosclerosis 2011;219:280-4.

8 Fadelu T, Zhang S, Niedzwiecki D, et al. Nut consumption and survival in patients with stage III colon cancer: results from CALGB 89803 (Alliance). J Clin Oncol 2018;36:1112-20.

9 Powers WJ, Rabinstein AA, Ackerson T, et al. 2018 guidelines for the early management of patients with acute ischemic stroke: a guideline for healthcare professionals from the American heart Association/ American stroke association. Stroke 2018;49:e46-110.

10 Brignole M, Moya A, de Lange FJ, et al. 2018 ESC guidelines for the diagnosis and management of syncope. Eur Heart $J$ 2018;39:ehy037:1883-948.

11 Salmerón J, Ascherio A, Rimm EB, et al. Dietary fiber, glycemic load, and risk of NIDDM in men. Diabetes Care 1997;20:545-50.

12 Salmerón J, Manson JE, Stampfer MJ, et al. Dietary fiber, glycemic load, and risk of non-insulin-dependent diabetes mellitus in women. JAMA 1997;277:472-7.

13 Ascherio A, Rimm EB, Hernán MA, et al. Intake of potassium, magnesium, calcium, and fiber and risk of stroke among US men. Circulation 1998;98:1198-204.

14 Iso $\mathrm{H}$, Stampfer MJ, Manson JE, et al. Prospective study of calcium, potassium, and magnesium intake and risk of stroke in women. Stroke 1999;30:1772-9.

15 Kao WH, Folsom AR, Nieto FJ, et al. Serum and dietary magnesium and the risk for type 2 diabetes mellitus: the Atherosclerosis risk in Communities study. Arch Intern Med 1999;159:2151-9.

16 Liu S, Manson JE, Stampfer MJ, et al. A prospective study of wholegrain intake and risk of type 2 diabetes mellitus in US women. Am J Public Health 2000;90:1409-15.

17 Meyer KA, Kushi LH, Jacobs DR, et al. Carbohydrates, dietary fiber, and incident type 2 diabetes in older women. Am J Clin Nutr 2000;71:921-30.

18 Hodge AM, English DR, O'Dea K, et al. Glycemic index and dietary fiber and the risk of type 2 diabetes. Diabetes Care 2004;27:2701-6.

19 Lopez-Ridaura R, Willett WC, Rimm EB, et al. Magnesium intake and risk of type 2 diabetes in men and women. Diabetes Care 2004;27:134-40.

20 Song Y, Manson JE, Buring JE, et al. Dietary magnesium intake in relation to plasma insulin levels and risk of type 2 diabetes in women. Diabetes Care 2004;27:59-65.

21 Song Y, Manson JE, Cook NR, et al. Dietary magnesium intake and risk of cardiovascular disease among women. Am J Cardiol 2005:96:1135-41.

22 Liu S, Choi HK, Ford E, et al. A prospective study of dairy intake and the risk of type 2 diabetes in women. Diabetes Care 2006;29:1579-84.

23 Pereira MA, Parker ED, Folsom AR. Coffee consumption and risk of type 2 diabetes mellitus: an 11-year prospective study of 28812 postmenopausal women. Arch Intern Med 2006;166:1311-6.

24 Pittas AG, Dawson-Hughes B, Li T, et al. Vitamin D and calcium intake in relation to type 2 diabetes in women. Diabetes Care 2006;29:650-6.

25 van Dam RM, Hu FB, Rosenberg L, et al. Dietary calcium and magnesium, major food sources, and risk of type 2 diabetes in U.S. black women. Diabetes Care 2006;29:2238-43.

26 Schulze MB, Schulz M, Heidemann C, et al. Fiber and magnesium intake and incidence of type 2 diabetes: a prospective study and meta-analysis. Arch Intern Med 2007;167:956-65.

27 Larsson SC, Virtanen MJ, Mars M, et al. Magnesium, calcium, potassium, and sodium intakes and risk of stroke in male smokers. Arch Intern Med 2008;168:459-65.

28 Weng L-C, Yeh W-T, Bai C-H, et al. Is ischemic stroke risk related to folate status or other nutrients correlated with folate intake? Stroke 2008:39:3152-8.
29 Kirii $\mathrm{K}$, Mizoue T, Iso H, et al. Calcium, vitamin D and dairy intake in relation to type 2 diabetes risk in a Japanese cohort. Diabetologia 2009;52:2542-50.

30 Ohira T, Peacock JM, Iso $\mathrm{H}$, et al. Serum and dietary magnesium and risk of ischemic stroke: the Atherosclerosis risk in Communities study. Am J Epidemiol 2009;169:1437-44.

31 Villegas R, Gao Y-T, Dai Q, et al. Dietary calcium and magnesium intakes and the risk of type 2 diabetes: the Shanghai women's health study. Am J Clin Nutr 2009;89:1059-67.

32 Hopping BN, Erber E, Grandinetti A, et al. Dietary fiber, magnesium, and glycemic load alter risk of type 2 diabetes in a multiethnic cohort in Hawaii. J Nutr 2010;140:68-74.

33 Kim DJ, Xun P, Liu K, et al. Magnesium intake in relation to systemic inflammation, insulin resistance, and the incidence of diabetes. Diabetes Care 2010;33:2604-10.

34 Kirii K, Iso H, Date C, et al. Magnesium intake and risk of selfreported type 2 diabetes among Japanese. J Am Coll Nutr 2010;29:99-106.

35 Nanri A, Mizoue T, Noda M, et al. Magnesium intake and type II diabetes in Japanese men and women: the Japan public health Center-Based prospective study. Eur J Clin Nutr 2010;64:1244-7.

36 Larsson SC, Virtamo J, Wolk A. Potassium, calcium, and magnesium intakes and risk of stroke in women. Am J Epidemiol 2011;174:35-43.

37 Weng L-C, Lee N-J, Yeh W-T, et al. Lower intake of magnesium and dietary fiber increases the incidence of type 2 diabetes in Taiwanese. $J$ Formos Med Assoc 2012;111:651-9.

38 Zhang W, Iso H, Ohira T, et al. Associations of dietary magnesium intake with mortality from cardiovascular disease: the JACC study. Atherosclerosis 2012;221:587-95.

39 Hata A, Doi Y, Ninomiya T, et al. Magnesium intake decreases type 2 diabetes risk through the improvement of insulin resistance and inflammation: the Hisayama study. Diabet Med 2013;30:1487-94.

40 Lin P-H, Yeh W-T, Svetkey LP, et al. Dietary intakes consistent with the DASH dietary pattern reduce blood pressure increase with age and risk for stroke in a Chinese population. Asia Pac J Clin Nutr 2013;22:482-91.

41 Oba S, Nanri A, Kurotani K, et al. Dietary glycemic index, glycemic load and incidence of type 2 diabetes in Japanese men and women: the Japan public health Center-Based prospective study. Nutr J 2013;12:165.

42 Sluijs I, Czernichow S, Beulens JWJ. Dietary electrolytes and risk of ischemic stroke. Eur J Prev Cardiol 2013;20:S76.

43 Hruby A, Meigs JB, O'Donnell CJ, et al. Higher magnesium intake reduces risk of impaired glucose and insulin metabolism and progression from prediabetes to diabetes in middle-aged Americans. Diabetes Care 2014;37:419-27.

44 Sluijs I, Czernichow S, Beulens JWJ, et al. Intakes of potassium, magnesium, and calcium and risk of stroke. Stroke 2014;45:1148-50.

45 Adebamowo SN, Spiegelman D, Flint AJ, et al. Intakes of magnesium, potassium, and calcium and the risk of stroke among men. Int J Stroke 2015;10:1093-100.

46 Adebamowo SN, Spiegelman D, Willett WC, et al. Association between intakes of magnesium, potassium, and calcium and risk of stroke: 2 cohorts of US women and updated meta-analyses. Am J Clin Nutr 2015;101:1269-77.

47 Bain LKM, Myint PK, Jennings A, et al. The relationship between dietary magnesium intake, stroke and its major risk factors, blood pressure and cholesterol, in the EPIC-Norfolk cohort. Int J Cardiol 2015;196:108-14.

48 Huang Y-C, Wahlqvist ML, Kao M-D, et al. Optimal dietary and plasma magnesium statuses depend on dietary quality for a reduction in the risk of all-cause mortality in older adults. Nutrients 2015;7:5664-83.

49 Hruby A, Guasch-Ferré M, Bhupathiraju SN, et al. Magnesium intake, quality of carbohydrates, and risk of type 2 diabetes: results from three U.S. cohorts. Diabetes Care 2017:40:1695-702.

50 Kokubo Y, Saito I, Iso H, et al. Dietary magnesium intake and risk of incident coronary heart disease in men: a prospective cohort study. Clin Nutr 2018;37:1602-8.

51 Konishi K, Wada K, Tamura T, et al. Dietary magnesium intake and the risk of diabetes in the Japanese community: results from the Takayama study. Eur J Nutr 2017;56:767-74.

52 Dong J-Y, Xun P, He K, et al. Magnesium intake and risk of type 2 diabetes: meta-analysis of prospective cohort studies. Diabetes Care 2011;34:2116-22.

53 Larsson SC, Orsini N, Wolk A. Dietary magnesium intake and risk of stroke: a meta-analysis of prospective studies. Am J Clin Nutr 2012;95:362-6.

54 Adams HP, Bendixen BH, Kappelle LJ, et al. Classification of subtype of acute ischemic stroke. definitions for use in a multicenter clinical 
trial. TOAST. Trial of Org 10172 in acute stroke treatment. Stroke 1993:24:35-41.

55 Rannikmäe K, Woodfield R, Anderson CS, et al. Reliability of intracerebral hemorrhage classification systems: a systematic review. Int J Stroke 2016;11:626-36.

56 Wells GA, Shea B, O'Connell D, et al. The Newcastle-Ottawa scale (NOS) for assessing the quality of Non-Randomized studies in metaanalysis. Appl Eng Agric 2014;18:727-34.

57 Higgins JPT, Thompson SG, Deeks JJ, et al. Measuring inconsistency in meta-analyses. BMJ 2003;327:557-60.

58 Greenland S, Longnecker MP. Methods for trend estimation from summarized dose-response data, with applications to meta-analysis. Am J Epidemiol 1992:135:1301-9.

59 Orsini N, Bellocco R, Greenland S. Generalized least squares for trend estimation of summarized dose-response data. Stata $J$ 2006;6:40-57.

60 Begg CB, Mazumdar M. Operating characteristics of a rank correlation test for publication bias. Biometrics 1994;50:1088-101.

61 Egger M, Davey Smith G, Schneider M, et al. Bias in meta-analysis detected by a simple, graphical test. BMJ 1997;315:629-34.

62 Manson JE, Bassuk SS. Vitamin and mineral supplements: what clinicians need to know. JAMA 2018;319:859-60.

63 Papanicolas I, Woskie LR, Jha AK. Health care spending in the United States and other high-income countries. JAMA 2018;319:1024-39.

64 Temprosa M, Diabetes Prevention Program Research Group. Long-Term effects of lifestyle intervention or metformin on diabetes development and microvascular complications over 15-year follow-up: the diabetes prevention program outcomes study. Lancet Diabetes Endocrinol 2015;3:866-75.

65 Maruthappu M, Sood H, Keogh B. Radically upgrading diabetes prevention in England. Lancet Diabetes Endocrinol 2015;3:312-3.

66 American Diabetes Association. 5. Prevention or Delay of Type 2 Diabetes: Standards of Medical Care in Diabetes-2018. Diabetes Care 2018;41:S51-4.

67 Guerrero-Romero F, Simental-Mendía LE, Hernández-Ronquillo $\mathrm{G}$, et al. Oral magnesium supplementation improves glycaemic status in subjects with prediabetes and hypomagnesaemia: a double-blind placebo-controlled randomized trial. Diabetes Metab 2015;41:202-7.

68 Ramadass S, Basu S, Srinivasan AR. SERUM magnesium levels as an indicator of status of diabetes mellitus type 2. Diabetes Metab Syndr 2015;9:42-5.

69 Eimerl S, Schramm M. The quantity of calcium that appears to induce neuronal death. J Neurochem 2010;62:1223-6.

70 van den Bergh WM, Algra A, van Kooten F, et al. Magnesium sulfate in aneurysmal subarachnoid hemorrhage: a randomized controlled trial. Stroke 2005;36:1011-5.

71 Goyal N, Tsivgoulis G, Malhotra K, et al. Serum magnesium levels and outcomes in patients with acute spontaneous intracerebral hemorrhage. J Am Heart Assoc 2018;7:e008698.

72 Willett WC, Howe GR, Kushi LH. Adjustment for total energy intake in epidemiologic studies. Am J Clin Nutr 1997;65:1220S-8. discussion 1229S-1231S.

73 Stone MS, Martyn L, Weaver CM. Potassium intake, bioavailability, hypertension, and glucose control. Nutrients 2016;8:E444.

74 Pittas AG, Lau J, Hu FB, et al. The role of vitamin D and calcium in type 2 diabetes. A systematic review and meta-analysis. J Clin Endocrinol Metab 2007;92:2017-29.

75 Larsson SC, Orsini N, Wolk A. Dietary calcium intake and risk of stroke: a dose-response meta-analysis. Am J Clin Nutr 2013;97:951-7.

76 Fang X, Wang K, Han D, et al. Dietary magnesium intake and the risk of cardiovascular disease, type 2 diabetes, and all-cause mortality: a dose-response meta-analysis of prospective cohort studies. BMC Med 2016;14:210

77 Fang X, Han H, Li M, et al. Dose-response relationship between dietary magnesium intake and risk of type 2 diabetes mellitus: a systematic review and meta-regression analysis of prospective cohort studies. Nutrients 2016;8:739.

78 Riddle MC, Ambrosius WT, Brillon DJ, et al. Epidemiologic relationships between $\mathrm{A} 1 \mathrm{c}$ and all-cause mortality during a median 3.4-year follow-up of glycemic treatment in the Accord trial. Diabetes Care 2010;33:983-90. 
Correction: Association of magnesium intake with type 2

diabetes and total stroke: an updated systematic review

and meta-analysis

Zhao B, Zeng L, Zhao J, et al. Association of magnesium intake with type 2 diabetes and total stroke: an updated systematic review and meta-analysis. BMJOpen 2020;10:e032240. doi: 10.1136/bmjopen-2019-032240

The affiliation for Binghao Zhao was incomplete in the published article. The correct affiliations are below.

Department of Cardio-Thoracic Surgery, The Second Affiliated Hospital of Nanchang University, Nanchang, Jiangxi, China.

Departments of Neurosurgery, Peking Union Medical College Hospital, Chinese Academy of Medical Sciences and Peking Union Medical College, China.

Open access This is an open access article distributed in accordance with the Creative Commons Attribution Non Commercial (CC BY-NC 4.0) license, which permits others to distribute, remix, adapt, build upon this work non-commercially, and license their derivative works on different terms, provided the original work is properly cited, appropriate credit is given, any changes made indicated, and the use is non-commercial. See: http://creativecommons.org/licenses/by-nc/4.0/.

C Author(s) (or their employer(s)) 2020. Re-use permitted under CC BY-NC. No commercial re-use. See rights and permissions. Published by BMJ.

BMJ Open 2020;10:e032240corr1. doi:10.1136/bmjopen-2019-032240corr1

Check for updates 